\title{
Design of Robust PID Controller Based on Desired Loop Transfer Function
}

\author{
Yonghui Chen \\ School of Automation and Electronic Information \\ Sichuan University of Science \& Engineering \\ Zigong, China \\ cyh0418@163.com
}

\author{
Gongquan Tan \\ School of Automation and Electronic Information \\ Sichuan University of Science \& Engineering \\ Zigong, China \\ tgq77@126.com
}

\begin{abstract}
Design method of loop transfer function on expected phase for robust gain system is proposed. Bode ideal loop function (BILF) can guarantee the strongly gain robust stability for closed loop system, while the desired fractional order (1 2) BILF has to be approximated with high integer order function to be implement. Low integer order controller, the conventional proportional integral and derivative (PID) controller, can compensate process function to be BILF on phase in mid-band frequency. So, the PID parameters can be obtained with phase fitting in appropriate frequency range. Examples are used to illustrate the proposed approach. Simulations show that this proposed method is effective for multiple lag and/or time delay processes.
\end{abstract}

Keywords-Bode ideal loop function; Robust control; Fractional order; PID controller; Expected phase

\section{INTRODUCTION}

Proportional, integral and derivative (PID) control is a basic control method for industrial processes. In recent 50 years, lots of PID tuning methods appears, wherein the PID controller design method based on the internal model principle has been gained widespread in recently [1]. However, the tradeoff parameter $\lambda$ of internal model controller is a still trouble thing for performance and robustness. An acceptable universal controller design approach does not been set up until now $[2,3]$.

Recently, successful applications of fractional order control systems had been reported in various engineering fields. Moreover, the focus problem in current is the research of parameter tuning or optimization of $\mathrm{PI}^{\lambda} \mathrm{D}^{\mu}$ controller proposed by Podlubny [4]. Monje et al. [5] established the experimental application for first-order plus delay time process (FOPDT) with the optimization of fractional order controller in the multi-objective constraints of crossover frequency, robust gain, and sensitivity and complement sensitivity limits. Chen et al [6] obtained a parameter tuning formula for fractional PI controller for FOPDT based on integral gain optimization with sensitivity function constraints. Luo et al [7] got the controller parameters based on directly solving three conditions of crossover frequency, phase margin and gain robustness. Rinku Singhal et al [8] tuned the coefficients $\lambda$ and $\mu$ of fractional order controller on the basis of the traditional integer order PID. The disadvantage of these methods is the need to implement the fractional order function with integer order function, especially accurate approximation result high integer order transfer function. Ramiro, etc. [9], and He Yiwen, etc. [4] tuned the controllers based on the optimization of various integral performance of error response of control system consisting of BILF, but these approaches do not guarantee the given phase margin of control systems [9].

In this work, a simple design method of robust PID parameters is proposed to solve above problems. This method first tune the integral and derivative time constant based on the phase characteristics of Bode idea loop function (BILF), and then, the integral gain is selected in order to determine the crossover frequency of loop function which is relevant of the response speed of the control system. At last, examples are used to illustrate this approach.

\section{IDEA LOOP FUNCTION AND ITS IMPLEMENTATION}

The structure of control system is shown in Figure 1, where $P(\mathrm{~s}), P_{L}(\mathrm{~s})$ and $C(\mathrm{~s})$ are the controlled process, the disturbance process and the controller respectively.

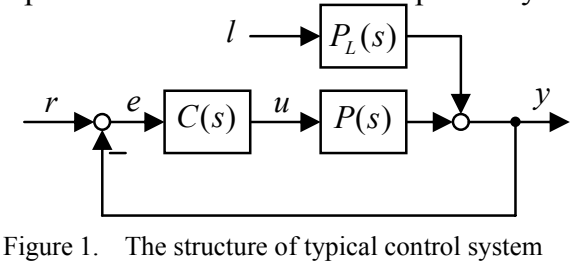

Bode proposed a desired open loop transfer function

$$
G(s)=P(s) C(s)=\left(\frac{\omega_{c}}{s}\right)^{\alpha}
$$

Where, $\omega_{c}$ is the crossover frequency of the system, $\alpha$ is the positive real number satisfying the requirements of system stability. The magnitude and phase of (1) are

$$
\left\{\begin{array}{l}
M_{G}(\omega)=|G(j \omega)|=\left(\frac{\omega_{c}}{\omega}\right)^{\alpha} \\
\varphi_{G}(\omega)=\angle G(j \omega)=-\frac{\alpha \pi}{2}
\end{array}\right.
$$

Obviously, the magnitude curve of Bode ideal loop function (BILF) is a line with slope $-20 \alpha \mathrm{dB} /$ decade, and the phase curve is $\mathrm{a}-\alpha \pi / 2$ radians horizontal line. When $0<\alpha<2$, gain margin of the closed-loop system is an infinite and the phase margin is $\mathrm{Pm}=\pi(1-\alpha / 2)$. This is why closed loop system has a strongly robustness to gain variation.

The expected value of $\alpha$ in BILF should be between 1 and 2, which means the corresponding system is a fractional 
order system. For implementation in practice, the fractional order function should be approximated by integer order function. Oustaloup proposed a method called OustaloupRecursive-Approximation (ORA) [10] to meet this case. That is, a fractional function can be approximated by a rational function as below

$$
H(s)=s^{\alpha} \approx \hat{H}(s)=K_{0} \prod_{k=-N}^{N} \frac{s+\omega_{k}^{\prime}}{s+\omega_{k}}
$$

Where, $\alpha \in[-1,1],\left[\omega_{b}, \omega_{h}\right]$ is a given frequency range and the zeros, poles and gain are:

$$
\left\{\begin{array}{l}
\omega_{k}^{\prime}=\omega_{b}\left(\frac{\omega_{h}}{\omega_{b}}\right)^{\frac{k+N+(1-\alpha) / 2}{2 N+1}} \\
\omega_{k}=\omega_{b}\left(\frac{\omega_{h}}{\omega_{b}}\right)^{\frac{k+N+(1+\alpha) / 2}{2 N+1}} \\
K_{0}=\left(\frac{\omega_{h}}{\omega_{b}}\right)^{-\frac{\alpha}{2}} \prod_{k=-N}^{N} \frac{\omega_{k}}{\omega_{k}^{\prime}}
\end{array}\right.
$$

For example, assume the desired loop function is $\mathrm{G}(\mathrm{s})=$ $s^{-0.5} / s$. With the frequency range $[0.01,100] \mathrm{rad} / \mathrm{s}, \mathrm{G}(\mathrm{s})$ was approximated by a rational function as in [11]

$$
G_{a}(s)=\frac{s^{5}+74.97 s^{4}+768.5 s^{3}+1218 s^{2}+298.5 s+10}{10 s^{5}+298.5 s^{4}+1218 s^{3}+768.5 s^{2}+74.97 s+1} \cdot \frac{1}{s}
$$
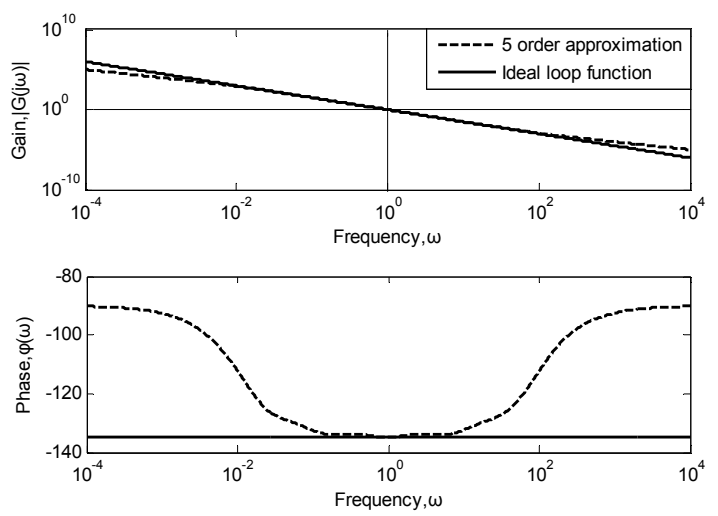

Figure 2. Bode ideal function and its rational approximation

The Bode plots of loop function $G(\mathrm{~s})$ and $G_{a}(\mathrm{~s})$ are shown in figure 2 by the solid line and the dashed line respectively. Obviously, the accepted approximation $G_{a}(\mathrm{~s})$ can play well within the given frequency range, while besides this range, there have distinct difference between $G(\mathrm{~s})$ and $G_{a}(\mathrm{~s})$ especially of its phase. So, designate the frequency boundaries are important for rational approximation from fractional function.

\section{EXPECTED CONTROL FUNCTION AND ITS PID APPROXIMATION}

Considering Bode ideal loop function as the controller design objectives, the expected control function can be expressed as

$$
C(s)=P^{-1}(s) G(s)=\frac{\omega_{c}^{\alpha}}{s^{\alpha} P(s)}
$$

Where, $\mathrm{G}(\mathrm{s})$ is Bode idea loop function and $\mathrm{P}(\mathrm{s})$ is the transfer function of process.

Choose the conventional PID controller as the apparatus; its function is expressed as

$$
\begin{gathered}
C_{\text {pid }}(s)=K_{c}\left(1+\frac{1}{T_{i} s}+T_{d} s\right) \\
=K_{i} \frac{1+B s+A s^{2}}{s}
\end{gathered}
$$

Where, $K_{i}=K_{c} / T_{i}, B=T_{i}, A=T_{d} T_{i}$.

The goal is to tune the PID from the expected control function. From (6) and (7), two facts can be found. First, PID function doesn't express the expected control function due to $\alpha>1$ at zero frequency, or extended to the low frequency range. Second, PID function doesn't express the expected control function at infinite frequency, or extended to the high frequency range, when $\mathrm{P}(\mathrm{s})$ has multiple lag and/or delay time. These two points can also be confirmed in figure 2 as previously demonstrated.

Therefore, the conventional PID approximation of idea control function must be in some medium frequency range like below

$$
C_{P I D}(j \omega) \approx C(j \omega),\left(\omega \in\left[\omega_{b}, \omega_{h}\right]\right)
$$

Further, the frequency range of the flat phase determines the gain robustness, that is, the phase approximation is more important than the magnitude approximation. For the same effect, literature [5] and [7] making the derivative of phase function as zero on the crossover frequency, that is, $\mathrm{d} \varphi_{G} / \mathrm{d} \omega\left(\omega=\omega_{c}\right)=0$. But, this condition can not guarantee the width of frequency range of flat phase. It should be better to made a given range for frequency requirement, that is

$$
\varphi_{P I D} \approx \varphi_{C}=\varphi_{G}-\varphi_{P},\left(\omega \in\left[\omega_{b}, \omega_{h}\right]\right)
$$

Where, $\varphi_{P I D}, \varphi_{C}, \varphi_{G}$ and $\varphi_{P}$ are the phase of PID controller, idea control function, Bode idea loop function and process respectively.

Although the phase range of conventional idea parallel PID is $\left(-90^{\circ}, 90^{\circ}\right)$, but the effective frequency can not extend to zero and infinite. Further more, if a process is consistent of multiple lag or delay factor, the controller would not compensate it up to some high frequency. So the phase approximation must be a narrow range, and a suitable phase range is refers to negative. Obviously, determine the phase range is more physical meaning than frequency range, and the frequency range $\left[\omega_{b}, \omega_{h}\right]$ can easily be solved from a given phase range $\left[\varphi_{b}, \varphi_{h}\right]$ according to the phase-frequency function $\varphi_{C}$ from idea control in (6). Thus, the nonlinear optimization problem can be expressed as 


$$
\begin{aligned}
\min _{A, B} J & =\int_{\omega_{b}}^{\omega_{h}}\left|\varphi_{p i d}(A, B, \omega)-\varphi_{C}(\omega)\right| d \omega \\
= & \int_{\omega_{b}}^{\omega_{h}}\left|\operatorname{tg}^{-1}\left(\frac{B \omega}{1-A \omega^{2}}\right)-\frac{\pi}{2}-\varphi_{C}(\omega)\right| d \omega
\end{aligned}
$$

This optimization can be solved from MATLAB command fmin. Because crossover frequency is independent of integral time and derivative time constants of PID, it can be tuned according to response requirement of system.

\section{Simulation}

Two examples were used to illustrate the approach.

\section{A. Example 1}

Multiple lag process $\mathrm{P}(\mathrm{s})[4,9]$, the selected Bode idea loop function $\mathrm{G}(\mathrm{s})$ and the solved idea control function $\mathrm{C}(\mathrm{s})$ from (6) are respectively as below:

$$
\left\{\begin{array}{l}
P(s)=\frac{1}{(s+1)^{3}} \\
G(s)=\left(\frac{\omega_{c}}{s}\right)^{1.5} \\
C(s)=P^{-1}(s) G(s)=\frac{\omega_{c}^{1.5}(s+1)^{3}}{s^{1.5}}
\end{array}\right.
$$

With the phase range $\left[-34^{\circ},-5.7^{\circ}\right]$, the corresponding frequency range $[0.6630,0.9353] \mathrm{rad} / \mathrm{s}$ can be solved according to the idea control function $C(\mathrm{j} \omega)$. Thus, optimizing (10) obtain $A=1.019$ and $B=1.244$. Select $\omega_{c}=1$ $\mathrm{rad} / \mathrm{s}$, this make integral gain $K_{i}=2.2144$ like as the same of [4] and [9]. By (7), the PID parameters are $K_{c}=2.7542, T_{i}=$ 1.2440 and $T_{d}=0.8191$. The Nyquist plot of proposed open loop system is as shown in Figure 3.

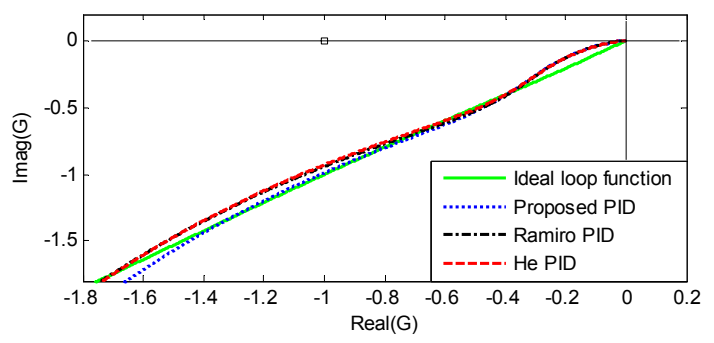

Figure 3. Nyquist plots of virous PID control systems

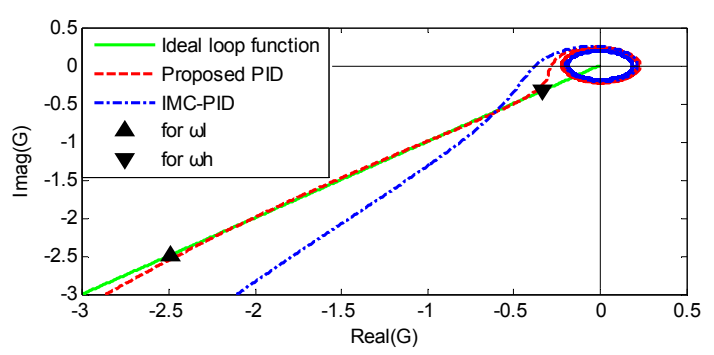

Figure 4. Nyquist plots of virous PID control systems
In figure 3, He Yiwen [4] and Ramiro [9] proposed control systems are also illustrated. Obviously, three cases are almost like. But it is noted that He-PID [4] and RamiroPID [9] require parameters searching in three-dimensional space, while the proposed method is only one time parameter fitting. The proposed operation is more simple and intuitive.

\section{B. Example 2}

Process feedback unit rig 38-100 device model as in [5], the selected Bode idea loop function G(s) and the solved idea control function $\mathrm{C}(\mathrm{s})$ from (6) are respectively as below:

$$
\left\{\begin{array}{l}
P(s)=\frac{3.13 e^{-50 s}}{433.33 s+1} \\
G(s)=\left(\frac{\omega_{c}}{s}\right)^{1.5} \\
C(s)=P^{-1}(s) G(s)=\frac{\omega_{c}^{1.5}}{3.13} \frac{(433.33 s+1) e^{50 s}}{s^{1.5}}
\end{array}\right.
$$

With the phase range $\left[-57.3^{\circ}, 0^{\circ}\right]$, the corresponding frequency range $[0.00475,0.0182] \mathrm{rad} / \mathrm{s}$ can be obtained through idea control function $\mathrm{C}(\mathrm{j} \omega)$. Thus, optimal parameters $A=2832, B=129.1$ are solved by MATLAB function fmin according to (10). Selecting $\omega_{c}=0.0110 \mathrm{rad} / \mathrm{s}$ makes integral gain $K_{i}=0.0108$ as the same as IMC-PID method $(\lambda=75)$ as in [12]. So, IMC-PID have the parameters $K_{c}=1.918, T_{i}=184.2$ and $T_{d}=13.56$, and proposed PID have parameters $K_{c}=1.388, T_{i}=129.1$ and $T_{d}=21.94$. Their Nyquist plots are shown in Figure 4. Its responses of closed loop system with set-point step at 0 second and disturbance step at 2000 seconds are shown in Figure 5. In these simulations, the perturbation system is the transfer function

$$
\tilde{P}=4.069 e^{-65 s} /(303.33 s+1)
$$

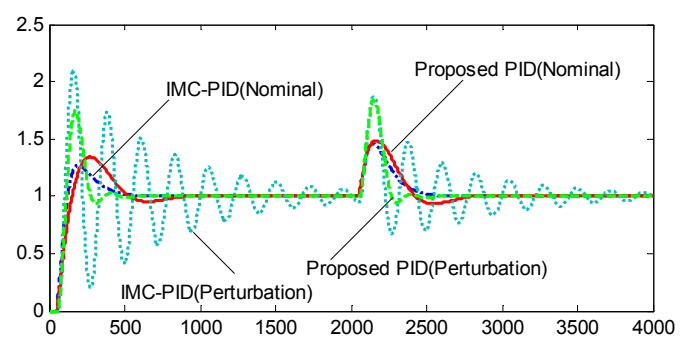

Figure 5. Step response of virous PID control systems

Figure 4 shows the proposed PID compensates process well to Bode idea loop function in mid-frequency range. Figure 5 shows, although set-point and disturbance response with IMC-PID is better than proposed method in nominal case, but the response of proposed approach is better than IMC-PID method in perturbation system which confirms the proposed PID has more robust performance on the system design expected.

\section{CONCLUSION}

A simple design method of robust PID controller based on approximation of ideal control function is proposed. The phase characteristic of the open loop transfer function 
adjusted by PID is to approximate the phase of Bode idea loop function. It retains the flat phase in mid-frequency range as possible as wide to guarantee the system has a strongly perturbation gain robustness. The proposed method can naturally attain the favorable range of frequency through the idea control function on given phase range and this decision is independent of the choice of crossover frequency, thus reducing the complexity of parameter tuning for controller and freely selecting the crossover frequency according to response speed requirement of system. Two design examples indicate that the recommended method can preserve the expected phase of Bode ideal loop function in mid-frequency range. Simulation results show the resulting control system has good robustness.

\section{ACKNOWLEDGMENT}

This work was supported by the Open Foundation of Artificial Intelligence Key Laboratory of Sichuan Province (Grant No. 2011RYJ07) and the Key Scientific Research Foundation of Education Department of Sichuan Province (Grant No. 12ZA097), China.

\section{REFERENCES}

[1] Ahmad Ali, Somanath Majhi, "PI/PID controller design based on IMC and percentage overshoot specification to controller setpoint change," ISA Transactions, vol. 48, Sep. 2008, pp. 10-15, doi: 10.1016/j.isatra.2008.09.002.

[2] K.J. Åström and T. Hägglund, Advanced PID Control. Instrument Society of America, North Carolina, 2006.

[3] Aidan O'Dwyer, PI and PID Controller Tuning Rules, $3^{\text {rd }}$ edtion. Imperial College Press, London, 2009.
[4] Yiwen He, Weisheng Xu, Yan Cheng, "Bode ideal transfer function in the fractional Control," Information and Control, vol. 39, No.2, April. 2010, pp. 200-206, doi: 1002-0411(2010)-02-0200-07.

[5] Concepción A. Monje, Blas M. Vinagre, Vicentr Feliu, YangQuan Chen, "Tuning and auto-tuning of fractional order controllers for industry applications," Control Engineering Practice, vol. 16, Aug. 2007, pp. 798-812, doi:10.1016/j.conengprac.2007.08.006.

[6] Yangquan Chen, Tripti Bhaskaran, and Dingyü Xue, "Practical tuning rules development for fractional order proportional and integral controllers," Journal of Computational and Nonlinear Dynamics, vol. 3, April 2008, pp.1-8, doi: 10.1115/1.2833934.

[7] Ying Luo, Yangquan Chen, Chunyang Wang, Youguo Pi, "Tuning fractional order proportional integral controllers for fractional order systems," Journal of Process Control, vol. 20, issue 7, April 2010, pp. 823-831, doi:10.1016/j.jprocont.2010.04.011.

[8] Rinku Singhal, Subhransu Padhee, Gagandeep Kaur, "Design of fractional order PID controller for speed control of DC motor," International Journal of Scientific and Research Publications, vol. 2, issue 6, June 2012, pp. 1-8.

[9] Ramiro S. Barbosa, J.A. Tenreiro Machado, and Isabel M. Ferreira, "Tuning of PID controllers based on Bode's ideal transfer function," Nonlinear Dynamics, vol. 38, issue 1-2, Mar. 2004, pp. 305-321.

[10] A. Oustaloup, F. Levron, B. Mathieu, F.M. Nanot, "Frequency -band complex non-integer differentiator: characterization and synthesis," IEEE Trans. On Circuits and Systems I: Fundamental Theory and Applications, vol. 47, issue 1, Jan. 2000, pp. 25-39.

[11] Ivo Petráš, "Fractional-order feedback control of a DC motor," Journal of Electical Engineering, vol. 60, issue 3, Mar. 2009, pp. 117128.

[12] Gongquan Tan, Yonghui Chen, Shuchuan Gan, "PID controller design based on modified IMC and optimal robust performance based on applied mechanics," Advanced Materials Research, vol. 485, Feb. 2012, pp. 114-118, doi:10.4028/www.scientific.net/AMR.485.114. 